Gut and Liver, Vol. 12, No. 4, July 2018, pp. 369-370

\title{
Effects of Antiplatelet Agents on Bleeding after Endoscopic Resection of Gastric Tumor
}

\author{
Ji Yong Ahn \\ Department of Gastroenterology, Asan Medical Center, University of Ulsan College of Medicine, Seoul, Korea
}

See "Continuous Use of Thienopyridine May Be as Safe as Low-Dose Aspirin in Endoscopic Resection of Gastric Tumors" by Sooyeon Oh, et al. on page 393-401, Vol. 12. No. 4, 2018

Endoscopic resection (ER) is accepted as an effective treatment for superficial gastric neoplasm without metastasis because of high rate of en bloc resection and low local recurrence rate. ${ }^{1}$ According to the safety and efficacy, the indications of ER has been widening in the area of therapeutic endoscopy. However, post-ER bleeding is one of the major complications after ER, and previous studies have reported that the use of antiplatelet agents is one of the risk factors for post-ER bleeding agents. ${ }^{2-5}$ As the use of antiplatelet agents has increased, the management of these drugs during perioperative period remains a matter of great concern. Patients receiving antiplatelet therapy have a potential risk of bleeding; therefore, withholding antiplatelet agents during the perioperative period of gastric ER has been thought to be essential. However, cessation of antiplatelet agents may lead to unexpected thrombotic events. Until now, many debating results about continuing or discontinuing antiplatelet therapy before gastric ER have been reported and there are no unified guidelines.

In this issue of Gut and Liver, Oh et al. ${ }^{6}$ retrospectively followed 215 patients who had performed gastric endoscopic submucosal dissection (ESD) between April 2005 and November 2016. The patients were exposed to antiplatelet therapy but not to anticoagulation and risk factors for bleeding such as the type of antiplatelet therapy, cessation period, and size of specimen were analyzed. Post-ESD bleeding rates were $13.5 \%$ in total patients, $12.8 \%$ in patients taking low-dose aspirin, 3.6\% in thienopyridine, $27.5 \%$ in dual antiplatelet agents, 33.3\% in triple agents, and 9.7\% in cessation group (withholding antiplatelet agents for 5-7 days before ESD) and 15.0\% in continuation group (for 0-4 days). Risk factors for bleeding after ESD were revealed as multiple antiplatelet therapy (odds ratio [OR], 2.41; 95\% confidence interval [CI], 1.01 to 5.76, $\mathrm{p}=0.049$ ) and specimen size $\geq 5.5 \mathrm{~cm}$ (OR, 2.84; CI, 1.04 to $7.73, \mathrm{p}=0.041)$ in multivariate analysis. However, there was no difference of bleeding risk according to the cessation period (OR, 1.83; CI 0.68 to 4.94 , $\mathrm{p}=0.231$ ) and statistically no significant between aspirin and thienopyridine users (OR, 0.23; CI, 0.05 to 1.09 , $\mathrm{p}=0.065$ ).

In this study, most of the results, such as multiple antiplatelet therapy and larger sized specimen increase the bleeding risk after gastric ER and thienopyridine is as safe as or safer than lowdose aspirin, agree with those of previous studies. However, the results about stopping antiplatelet agents is not correspond with the recent guideline by Asian Pacific association and society, which recommended interruption of all antithrombotic agents for ultra-high risk procedures such as ESD. ${ }^{7}$ There were conflicting previous reports as to whether continuing antithrombotic therapy increases bleeding after gastric ER or not and these retrospective observational studies reported either no increased risk $^{5,8}$ or an increased risk of bleeding. ${ }^{4}$ Even in guidelines, they recommend differently by the East and the West. American and European guidelines ${ }^{9,10}$ recommend continuing low-dose aspirin during high-risk endoscopic procedures. In contrast, Asian $\mathrm{Pa}-$ cific groups recently recommended stopping of antithrombotic agents before the procedures by classifying the endoscopic mucosal resection of polyps $>2 \mathrm{~cm}$ and ESD as ultra-high risk procedures, reflecting the specificity of Asians. ${ }^{7}$ The reasons about the difference of bleeding risk according to the use of antithrombotic agents between the East and West are not clear until now and, due to the many various and unpredictable situations in real clinical setting, well designed randomized case-

Correspondence to: Ji Yong Ahn

Department of Gastroenterology, Asan Digestive Disease Research Institute, Asan Medical Center, University of Ulsan College of Medicine, 88 Olympic-ro 43-gil, Songpa-gu, Seoul 05505, Korea

Tel: +82-2-3010-5667, Fax: +82-2-476-0824, E-mail: ji110@hanmail.net pISSN 1976-2283 eISSN 2005-1212 https://doi.org/10.5009/gnl18193

@ This is an Open Access article distributed under the terms of the Creative Commons Attribution Non-Commercial License (http://creativecommons.org/licenses/by-nc/4.0) which permits unrestricted non-commercial use, distribution, and reproduction in any medium, provided the original work is properly cited. 
control study is not performed in any study. In addition, as authors said, most of previous retrospective studies showed their own definition about cessation period and did not have a control group of non-antithrombotic agent users.

This is why this study is meaningful because the authors analyzed carefully about the risk of post-ER bleeding according to the antiplatelet use and these kinds of study are not enough due to the limitation of study design. Based on these results, further well designed studies are expected to make clinically helpful guidelines in this field.

\section{CONFLICTS OF INTEREST}

No potential conflict of interest relevant to this article was reported.

\section{REFERENCES}

1. Ahn JY, Jung HY, Choi KD, et al. Endoscopic and oncologic outcomes after endoscopic resection for early gastric cancer: 1370 cases of absolute and extended indications. Gastrointest Endosc 2011;74:485-493.

2. Kono Y, Obayashi Y, Baba Y, et al. Postoperative bleeding risk after gastric endoscopic submucosal dissection during antithrombotic drug therapy. J Gastroenterol Hepatol 2018;33:453-460.

3. Takeuchi T, Ota K, Harada S, et al. The postoperative bleeding rate and its risk factors in patients on antithrombotic therapy who undergo gastric endoscopic submucosal dissection. BMC Gastro- enterol 2013:13:136.

4. Cho SJ, Choi IJ, Kim CG, et al. Aspirin use and bleeding risk after endoscopic submucosal dissection in patients with gastric neoplasms. Endoscopy 2012;44:114-121.

5. Dong J, Wei K, Deng J, et al. Effects of antithrombotic therapy on bleeding after endoscopic submucosal dissection. Gastrointest Endosc 2017;86:807-816

6. Oh S, Kim SG, Kim J, et al. Continuous use of thienopyridine may be as safe as low-dose aspirin in endoscopic resection of gastric tumors. Gut Liver 2018;12:393-401.

7. Chan FKL, Goh KL, Reddy N, et al. Management of patients on antithrombotic agents undergoing emergency and elective endoscopy: joint Asian Pacific Association of Gastroenterology (APAGE) and Asian Pacific Society for Digestive Endoscopy (APSDE) practice guidelines. Gut 2018;67:405-417.

8. Tounou S, Morita Y, Hosono T. Continuous aspirin use does not increase post-endoscopic dissection bleeding risk for gastric neoplasms in patients on antiplatelet therapy. Endosc Int Open 2015;3:E31-E38.

9. ASGE Standards of Practice Committee, Acosta RD, Abraham NS, et al. The management of antithrombotic agents for patients undergoing GI endoscopy. Gastrointest Endosc 2016;83:3-16.

10. Veitch AM, Vanbiervliet G, Gershlick AH, et al. Endoscopy in patients on antiplatelet or anticoagulant therapy, including direct oral anticoagulants: British Society of Gastroenterology (BSG) and European Society of Gastrointestinal Endoscopy (ESGE) guidelines. Gut 2016;65:374-389. 